\title{
Screening strategies for active tuberculosis: focus on cost-effectiveness
}

\author{
Claudia Caroline Dobler \\ Clinical Management Group, \\ Woolcock Institute of Medical \\ Research, University of Sydney, \\ Sydney, NSW, Australia
}

This article was published in the following Dove Press journal:

ClinicoEconomics and Outcomes Research

30 June 2016

Number of times this article has been viewed

\begin{abstract}
In recent years, there has been renewed interest in screening for active tuberculosis (TB), also called active case-finding (ACF), as a possible means to achieve control of the global TB epidemic. ACF aims to increase the detection of TB, in order to diagnose and treat patients with TB earlier than if they had been diagnosed and treated only at the time when they sought health care because of symptoms. This will reduce or avoid secondary transmission of TB to other people, with the long-term goal of reducing the incidence of TB. Here, the history of screening for active TB, current screening practices, and the role of TB-diagnostic tools are summarized and the literature on cost-effectiveness of screening for active TB reviewed. Cost-effectiveness analyses indicate that community-wide ACF can be cost-effective in settings with a high incidence of TB. ACF among close TB contacts is cost-effective in settings with a low as well as a high incidence of TB. The evidence for cost-effectiveness of screening among HIV-infected persons is not as strong as for TB contacts, but the reviewed studies suggest that the intervention can be cost-effective depending on the background prevalence of TB and test volume. None of the cost-effectiveness analyses were informed by data from randomized controlled trials. As the results of randomized controlled trials evaluating different ACF strategies will become available in future, we will hopefully gain a better understanding of the role that ACF can play in achieving global TB control.
\end{abstract}

Keywords: disease elimination, epidemic, active case-finding, review

\section{Epidemiology of tuberculosis and role of screening}

Tuberculosis (TB) remains a leading cause of morbidity and mortality globally, predominantly affecting low- and middle-income countries. In 2013, an estimated 9 million people developed TB, and 1.5 million died from the disease. ${ }^{1}$ In 2014 , the World Health Organization (WHO) developed a post-2015 global TB strategy with a vision to progress toward TB elimination. ${ }^{2}$ The goals are a $95 \%$ reduction in TB deaths and a 90\% reduction in TB-incidence rate (fewer than ten TB cases per 100,000 population) from 2015 to 2035, and full elimination of TB (defined as under one case per million people per year) by 2050 . While the worldwide TB incidence decreased at an average rate of $\sim 1.5 \%$ per year between 2000 and 2013, TB-control efforts must be accelerated if the post-2015 targets are to be met. ${ }^{1}$

Strategies to improve TB control include: 1) treating every person in the population diagnosed with active TB with short-course directly observed treatment, a strategy strongly recommended by the $\mathrm{WHO}^{3}$ 2) reducing time to treatment; 3 ) increasing treatment success; 4) increasing the TB case-detection rate through passive case-finding

Correspondence: Claudia Dobler Clinical Management Group, Woolcock Institute of Medical Research, University of Sydney, 43I Glebe Point Road, Glebe, Sydney, NSW 2037, Australia

Tel +6I 291140000

Email cdobler@med.usyd.edu.au 
(PCF), which requires that patients are aware of their symptoms, have access to health facilities, and are evaluated by health workers or volunteers who recognize the symptoms of TB and who have access to a reliable laboratory; 5) TB screening/active $\mathrm{CF}$ (ACF) among target populations; and 6) preventive treatment among TB contacts and other highrisk groups, especially in countries with a low TB incidence. Screening for active TB, also called ACF, aims to increase the detection of TB cases among a specific population, in order to diagnose and treat patients with active TB earlier than if they had been diagnosed and treated only at the time when they sought health care (if at all) because of symptoms (PCF). Therefore, screening for active TB will reduce or avoid secondary transmission of TB to other people, with the longterm goal of reducing the incidence of TB in a specific setting. ACF entails screening through an outreach program outside established health-service facilities. Enhanced CF includes activities that raise awareness about TB symptoms among a population and promote self-presentation to medical services.

In recent years, there has been renewed interest in ACF as a possible means to achieve control of the global TB epidemic, as traditional TB-control strategies (increasing case-detection rate by PCF and using short-course directly observed treatment to increase treatment success) failed to reduce the long-term incidence of TB significantly. ${ }^{4} \mathrm{~A}$ modeling study found that in areas where a target case-detection rate of $70 \%$ and a treatment-success rate of $85 \%$ have already been achieved, maintaining stable case-detection levels may not meaningfully reduce TB incidence further. ${ }^{5}$ The study results suggested that case-detection targets above $70 \%$ must be pursued, in order to achieve TB elimination with existing tools. This paper summarizes strategies for ACF for TB, examining the evidence for TB screening among different target groups based on cost-effectiveness analyses (CEAs).

\section{Overview of different screening strategies for detection of active TB Historical perspective}

Screening for active TB can either include the whole population (mass screening) or it can focus on selected high-risk groups (targeted screening). In the mid-20th century, mass-screening campaigns were conducted in Europe and North America. Screening was performed using mass miniature radiography (MMR), first developed in 1936, using a miniature (50-100 $\mathrm{mm}$ ) photograph from a chest X-ray displayed on a fluorescent screen. MMR costs less than a large chest X-ray, and radiation exposure for patient and operator is less compared to standard chest X-rays. MMR played an important role during the Second World War (1939-1945) for screening of military personnel and civilians, ${ }^{6-8}$ and was subsequently scaled up when mobile MMR using "TB vans" became available, taking the screening out into the community, and thus greatly increasing the capacity for targeted and whole-community screening. ${ }^{9}$ While MMR has been successful in detecting previously unknown TB cases and diagnosing TB cases earlier, it remains unclear how much of the major reduction in TB incidence in North America and Europe in the 20th century can be attributed to this intervention, as TB incidence was already decreasing in developed countries in the first half of the 20th century because of better nutrition and housing conditions. Additionally, the introduction of effective anti-TB medication in the 1940s-1960s led to a steep decline in TB-related mortality and an accelerated drop in incidence of TB.

The financial cost and logistics associated with mass screening using MMR made it difficult to implement in resource-poor countries. ${ }^{10}$ In the 1960 s, new CF strategies, relying more on the detection of symptomatic patients, were trialed in developing countries. ${ }^{11}$ A seminal Indian study published in 1963 concluded that TB-control programs should primarily be based on PCF, as the majority of patients with bacteriologically confirmed TB had at least one major TB-related symptom. ${ }^{12}$ ACF strategies were proposed as an additional measure only, once the health care system was satisfactorily developed to take care of patients who sought medical attention for TB-related symptoms. Several studies suggested that focusing screening on people with a history of cough as a determining symptom was sufficient, and extensive history-taking was not required. ${ }^{13-15}$

Emphasis was thus put on developing a strong health system infrastructure with high-quality diagnostic services and increasing awareness of TB in health care workers. Large-scale studies from Europe, Canada, and Japan in the 1960s and early 1970s found that most cases of TB were detected through PCF and not periodic MMR screening. ${ }^{16-19}$ Based on these findings, the ninth report of the WHO Expert Committee on Tuberculosis in 1974 recommended that MMR be abandoned. ${ }^{20}$ The principles of the current WHO strategy of TB control were established, focusing on PCF and delivery of TB case-management activities through the general health infrastructure. ${ }^{10}$

\section{Community-wide screening in more recent times}

Despite the focus on PCF promoted by the WHO since 1974, there have been more recent studies that have evaluated ACF strategies in the whole population of a community 
or district. Screening algorithms and diagnostic tools used have varied, with sputum-smear microscopy in patients with a history of cough being a common screening tool. A systematic review that included studies published between 1980 and 2010 assessed the evidence that screening for TB: 1) increases the number of TB cases detected and initiated on anti-TB medication, 2) identifies cases at an earlier stage of disease, 3 ) reduces TB-related mortality and morbidity, and 4) impacts on TB epidemiology (incidence and prevalence of TB in the community) compared to PCF alone. ${ }^{21,22}$ The review identified four randomized trials ${ }^{23-26}$ and 14 prevalence studies that investigated the effect of screening on TB CF. An additional 14 studies provided data on the contribution of screening to the total TB cases diagnosed. The review found moderate evidence to suggest that screening increases the number of TB cases found in the short term. In many settings, more than half the prevalent TB cases in the community were undiagnosed. The risk of false-positive TB diagnosis, however, which is higher in populations undergoing ACF compared to PCF (due to the inverse relationship between false-positive diagnosis and TB prevalence) needs to be considered. There was moderate evidence that screening identifies TB cases earlier and with less severe disease, based on 15 studies from both high and low-TB-incidence settings. No significant impact of screening on TB-treatment outcomes was found based on a review of 17 studies, with similar treatment success in TB cases found through screening and PCF in varied settings with different proportions of successful treatment. Evidence that screening (in addition to PCF) impacts on TB epidemiology (incidence and prevalence of TB in the community) was weak, based on five studies. ${ }^{27-31}$ There were some significant study limitations, including lack of a control group without an intervention, ${ }^{27}$ methodological concerns about the calculation of the standardized TBnotification ratio, ${ }^{28}$ lack of long-term trends in TB incidence and unclear pairing of communities for randomization, ${ }^{29}$ and study assessment not limited to the effect of screening alone. ${ }^{30}$ ZAMSTAR was the only study with a clusterrandomized design that directly evaluated the impact of screening on TB epidemiology. It was published in 2013, and preliminary results only were available at the time of the systematic review. ${ }^{31}$ The study, conducted in Zambia and South Africa, used a $2 \times 2$ factorial design comparing enhanced $\mathrm{CF}$, a household intervention (combined TB-HIV activities based on the activities included in the WHO guidelines for collaborative TB-HIV care in clinics), both, or neither. Neither intervention led to a statistically significant reduction in TB. In summary, the evidence to support community-wide ACF in high-incidence settings is insufficient. Current WHO guidelines include a conditional recommendation for community-wide systematic screening for active TB, suggesting that screening "may be considered for geographically defined subpopulations with extremely high levels of undetected TB (1\% prevalence or higher)". ${ }^{32}$ Additionally, the guidelines propose that subpopulations with limited access to health services may be considered for TB screening. This includes people living in urban slums or remote areas, homeless people and other vulnerable or disadvantaged groups such as some indigenous populations, migrants and refugees.

A cluster-randomized controlled trial is under way in Vietnam that will evaluate the effect of yearly communitywide TB screening for 3 years compared to no screening on the prevalence of microbiologically confirmed pulmonary TB (measured in the intervention and control clusters in the fourth year). Residents are visited in their home, asked about symptoms of TB, and asked to produce a single spontaneous sputum specimen for testing. The collected sputum specimen will be tested for Mycobacterium tuberculosis using a fully automated polymerase chain reaction test (Xpert MTB/RIF; Cepheid Inc, Sunnyvale, CA, USA).

\section{Screening for active TB in high-risk groups}

The WHO's 2013 guidelines on systematic screening for active TB include strong recommendations for screening of: 1) household contacts and other close contacts of patients with active TB, 2) people living with HIV, and 3) current and former workers in workplaces with silica exposure. ${ }^{32}$ It is however worth noting that the guidelines state that direct evidence underlying these strong recommendations was poor, and the recommendations were mainly influenced by the views of the members of the guideline development group.

A strong recommendation in these guidelines was defined as one "for which the desirable effects of adhering to the recommendation are judged to clearly outweigh the undesirable effects (and for which) screening is judged to be feasible, acceptable, and affordable in all settings".

A Cochrane review published in 2011 examined whether ACF among contacts of people with confirmed TB increased case detection compared to usual practice. ${ }^{33}$ The review identified only one randomized controlled trial that tested 
the effect of ACF in contacts, but the intervention in that trial also included screening for and treatment of latent TB infection (LTBI) in contacts, and the separate effect of ACF could not be estimated. ${ }^{29}$ The ZAMSTAR study did not find a significant benefit of household screening in a clusterrandomized trial. ${ }^{31}$ The results of a pragmatic stepped-wedge cluster-randomized trial of ACF among household contacts of sputum smear-positive TB cases in Lima, Peru are pending. ${ }^{34}$ Also pending are the results of a cluster-randomized trial of ACF among household contacts of sputum smear-positive TB cases in Vietnam. ${ }^{35}$

The evidence for screening people living with HIV and current and former workers in workplaces with silica exposure is weak (despite strong recommendations by the WHO for screening), due to a lack of randomized controlled trials comparing ACF versus PCF in these high-risk groups. The WHO additionally outlines conditional screening recommendations for prisoners, people with an untreated fibrotic chest X-ray lesion, and people who are seeking health care or who are in health care if they belong to selected high-risk groups, such as patients with diabetes mellitus or patients with chronic renal failure or on hemodialysis. ${ }^{32}$

\section{Practical considerations}

A good screening test is characterized by high performance (sensitivity, specificity, and reproducibility) and favorable operational characteristics, including the time taken to perform the test, its technical simplicity or ease of use, user acceptability, and the stability of the test under the expected conditions of use. ${ }^{36}$ The ease of use will depend on how easy it is to acquire and maintain the equipment required to perform the test, how difficult it is to train staff to use the test and to interpret the results of the test correctly, and the stability of the test under the expected conditions of use. Until recently, the lack of rapid and accurate diagnostic tools for TB has been a major obstacle to achieving global TB control. ${ }^{37} \mathrm{~TB}$ diagnosis, even today, is still reliant on older diagnostic tools, such as direct smear microscopy and culture, tests which often perform poorly and for which the infrastructure is frequently unavailable in the periphery of the health system. Sputumsmear microscopy has been the primary method for diagnosis of pulmonary TB in low- and middle-income countries, but it has some significant limitations. Its sensitivity is highly variable, ranging between $20 \%$ and $80 \%,{ }^{38}$ with the lowest sensitivity in children ${ }^{39}$ and HIV-infected patients. ${ }^{40}$ Serial sputum examinations are required to increase sensitivity, but some patients do not return for repeated sputum examinations and become "diagnostic defaulters", ie, patients who do not complete the diagnostic process. ${ }^{41}$ Most of the diagnostic defaulters are unaware that they have a positive sputum smear and are thus infectious. Additionally, smear microscopy relies on well-trained microscopists: sensitivities can vary up to $28 \%$ between readings in the field and reference laboratories. ${ }^{42}$ The sensitivity of smear microscopy for extrapulmonary specimens is very low, because the specimens usually contain only a few irregularly distributed organisms that tend to clump together. ${ }^{43}$

In the last few years, new TB diagnostics have been introduced. The most significant shift in the TB-diagnostics landscape has been the worldwide rollout of Xpert MTB/ RIF. $^{44}$ The Xpert MTB/RIF is a cartridge-based automated diagnostic test that can identify $M$. tuberculosis DNA and resistance to rifampicin by polymerase chain reaction. The Xpert technology has significantly increased sensitivity for detection of TB compared to sputum-smear microscopy, and can also rapidly detect rifampicin resistance with high accuracy. ${ }^{45}$ Rifampicin resistance is highly indicative of multidrug-resistant (MDR) TB, although formal confirmation of isoniazid resistance is required. The WHO endorsed Xpert MTB/RIF in 2010 for use in TB-endemic countries, and declared it a major milestone for global TB diagnosis. According to the WHO, as of December 31, 2014, 3,763 GeneXpert instruments (comprising 17,883 modules) and $>10$ million Xpert MTB/RIF cartridges had been procured by the public sector in 116 of 145 countries eligible for concessional pricing. ${ }^{46}$ The worldwide success and rollout of MTB/RIF has contributed to considerable interest in new TB diagnostics. A significant expansion of molecular technologies that that could potentially replace smear microscopy can be expected. ${ }^{47}$ Unmet needs in TB diagnostics relevant to TB screening include: 1) a simple triage test to identify individuals with presumed TB who need confirmatory testing (currently used tools are symptom check, eg, cough for 2 weeks, which lacks sensitivity and specificity, and chest X-ray, which has low specificity for TB), 2) a sputum-based replacement test for smear microscopy (for diagnosis of active pulmonary TB); and 3) a nonsputum-based biomarker test for all forms of extrapulmonary TB, ideally suitable for use at levels below microscopy centers (for diagnosis of extrapulmonary and childhood TB). ${ }^{48}$

It is important to distinguish tests to detect active TB from tests to detect latent (dormant) TB infection. In most infected persons, TB remains clinically asymptomatic and microbiologically inactive (latent). Approximately 5\% of infected persons will progress from infection to primary TB disease, and another estimated $5 \%$ of people with LTBI 
will develop active TB through endogenous reactivation of LTBI at some point in their life (postprimary TB), sometimes many years after the initial infection. ${ }^{49}$ There are two available investigations for identification of LTBI: the tuberculin skin test (TST), also called the Mantoux test, and IFN $\gamma$-release assays (IGRAs). If a patient has a positive TST or IGRA, active TB needs to be ruled out (by asking about TB symptoms, performing chest X-ray and sputum smear if indicated) before preventive TB treatment can be started. A positive TST or IGRA result does not discriminate between LTBI and active TB disease. There is some concern that TSTs and IGRAs are being misused in some high-TB-incidence settings, such as India, to diagnose active TB. ${ }^{50}$ This will lead to significant overdiagnosis of active TB, as LTBI is highly prevalent in India. ${ }^{51}$

\section{Cost-effectiveness of screening strategies for active TB}

CEA is increasingly used to inform decisions on allocation of health care resources, including resources in TB care. It compares the costs and outcomes (effects) of two or more courses of action to assess the extent to which an intervention can be regarded as providing value for money. Cost-utility analysis is a form of CEA where effectiveness is expressed in terms of utility (quality-adjusted life years [QALYs]). In order to calculate QALYs, health-state utility scores need to be incorporated into a model. A utility score, obtained through preference-measurement techniques, reflects the "value" people place on a health state on a scale from 0 (equal to death) to 1 (equal to perfect health). Expressing effectiveness in terms of utility allows comparison across different health programs and policies by using a common unit of measure (cost/QALY gained). As an alternative to QALYs, disabilityadjusted LYs (DALYs) can be used in cost-utility analyses (cost/DALY avoided). DALYs are a measure of overall disease burden, expressed as the number of years lost due to ill-health, disability, or early death. For DALYs, the scale used to measure health state is inverted to a "severity scale", whereby 0 equates to perfect health and 1 equates to death. The weight factors are age-adjusted, and they are discounted with time, thus favoring immediate over future health benefits. ${ }^{52}$ While QALYs and DALYs both allow for comparison of interventions across health sectors, they are not interchangeable. The WHO recommends that DALYs are used to express health-outcome effectiveness, but states that QALYs can be used alternatively. ${ }^{53}$ If the effectiveness measure is diseasespecific (eg, cost per case of TB detected or per case of TB cured), comparison is only possible across interventions for this particular disease, and there is no standardized measure of what constitutes good value for money.

When (mutually exclusive) choices have to be made between interventions (or an intervention and no intervention) for the same health issue, eg, different screening strategies to detect active TB, the question that needs to be addressed is: What are the additional benefits to be gained from a new intervention, and at how much additional cost? The use of incremental cost-effectiveness ratios (ICERs) answers these questions. These are calculated by dividing the difference in costs (between interventions) by the difference in health effects (between interventions). In order to determine whether an intervention represents good value for a national health care system, the Commission on Macroeconomics and Health has proposed thresholds based on the per capita gross domestic product (GDP). ${ }^{54}$ Using this approach, promoted by the $\mathrm{CHOosing}$ Interventions that are Cost-Effective (WHO-CHOICE) project, an intervention that costs less than three times the national annual GDP per capita per DALY avoided is considered cost-effective, whereas one that costs less than once the national annual GDP per capita is considered highly cost-effective. ${ }^{55}$

To examine the available evidence on cost-effectiveness of screening for active TB, the author performed an electronic search on PubMed/Medline on March 6, 2016. I searched using the terms (screening OR case finding OR case-finding) AND (tuberculosis OR TB) AND (cost OR value). I included every study that assessed the cost-effectiveness of a screening intervention for active TB, compared with "doing nothing", ie, $\mathrm{PCF}$, or compared with other screening interventions for active TB. I excluded: 1) studies that focused on screening for LTBI or tests used primarily to detect LTBI (TST, IGRAs); 2) studies that included LTBI treatment for patients with abnormal chest $\mathrm{X}$-rays, but no active disease, in the overall cost-effectiveness assessment; 3 ) studies that compared only screening for active TB with screening for LTBI, but not to "no screening"; and 4) reviews or opinion pieces (editorials, letters, etc). Screening for LTBI with the intention to offer preventive TB treatment always incorporates screening for active TB, as active TB needs to be excluded before preventive TB treatment is initiated.

The search strategy identified 86 publications, of which 14 met the inclusion and exclusion criteria; one additional study was identified from the reference list of a paper (Table 1). ${ }^{56-71}$ The 15 identified studies contained 17 analyses of target groups for screening. Thirteen analyzes used ICERs, thus evaluating the difference in cost between two possible interventions (or an intervention and no intervention) divided by the difference in their effect. Four studies reported only the 


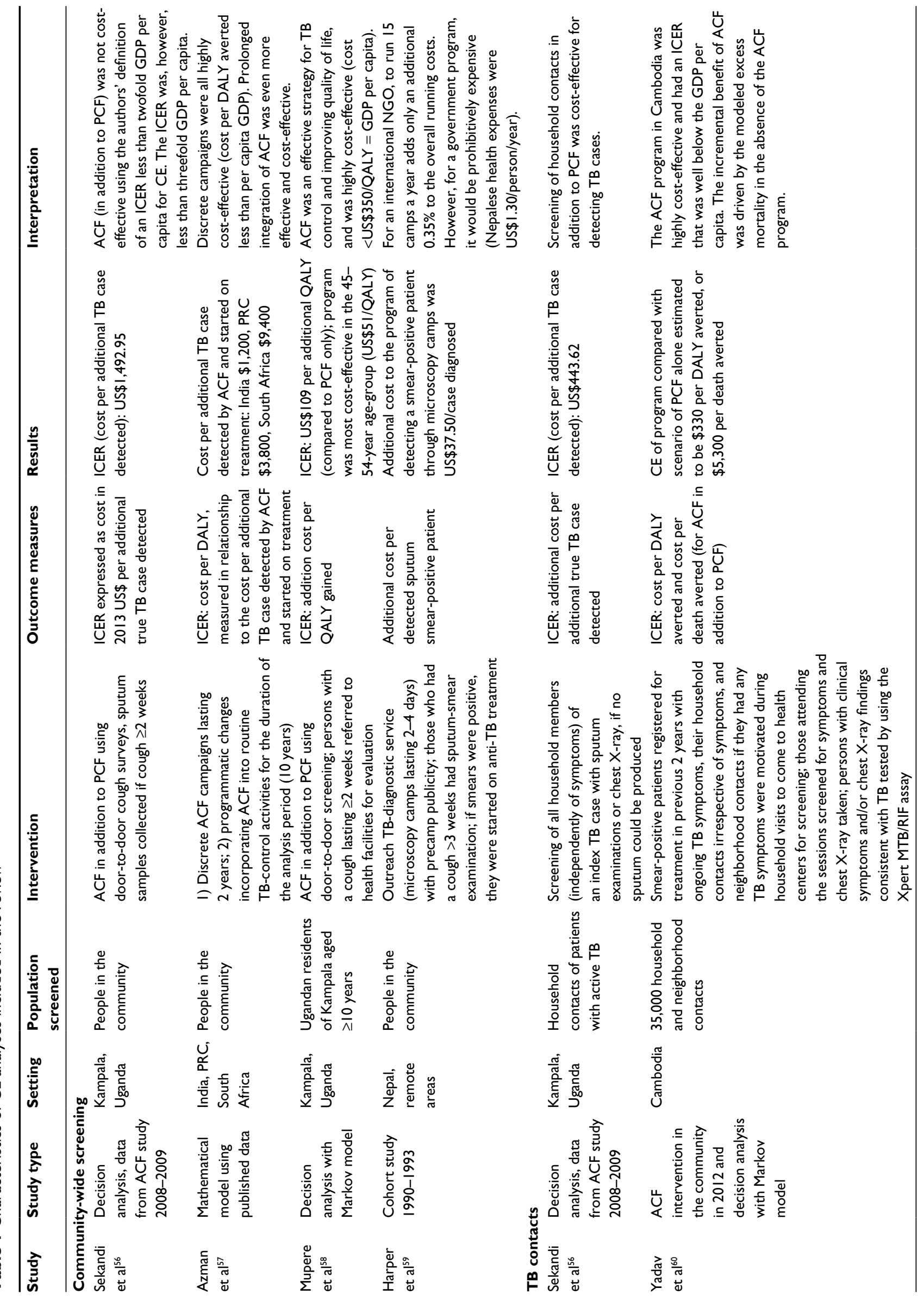




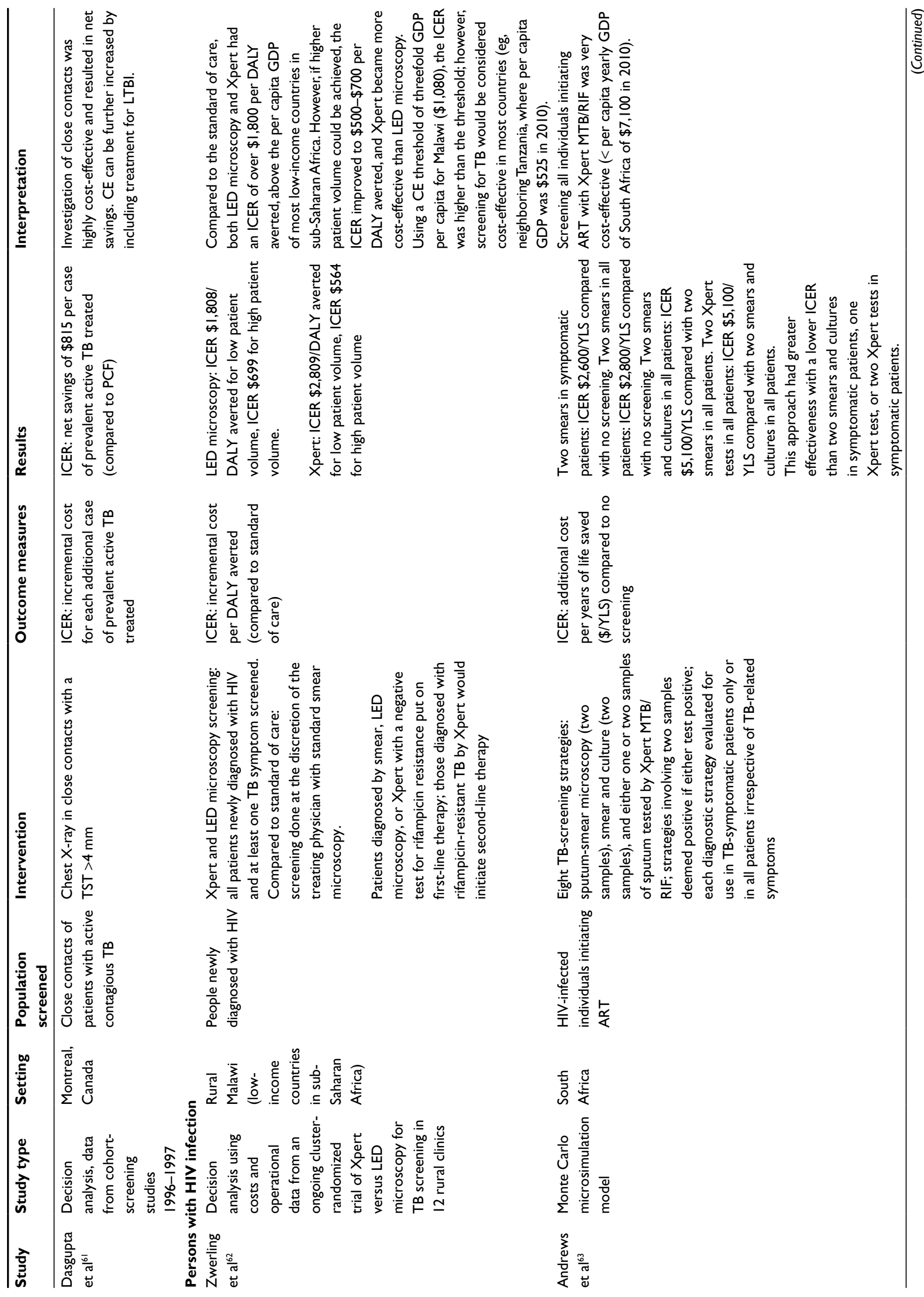




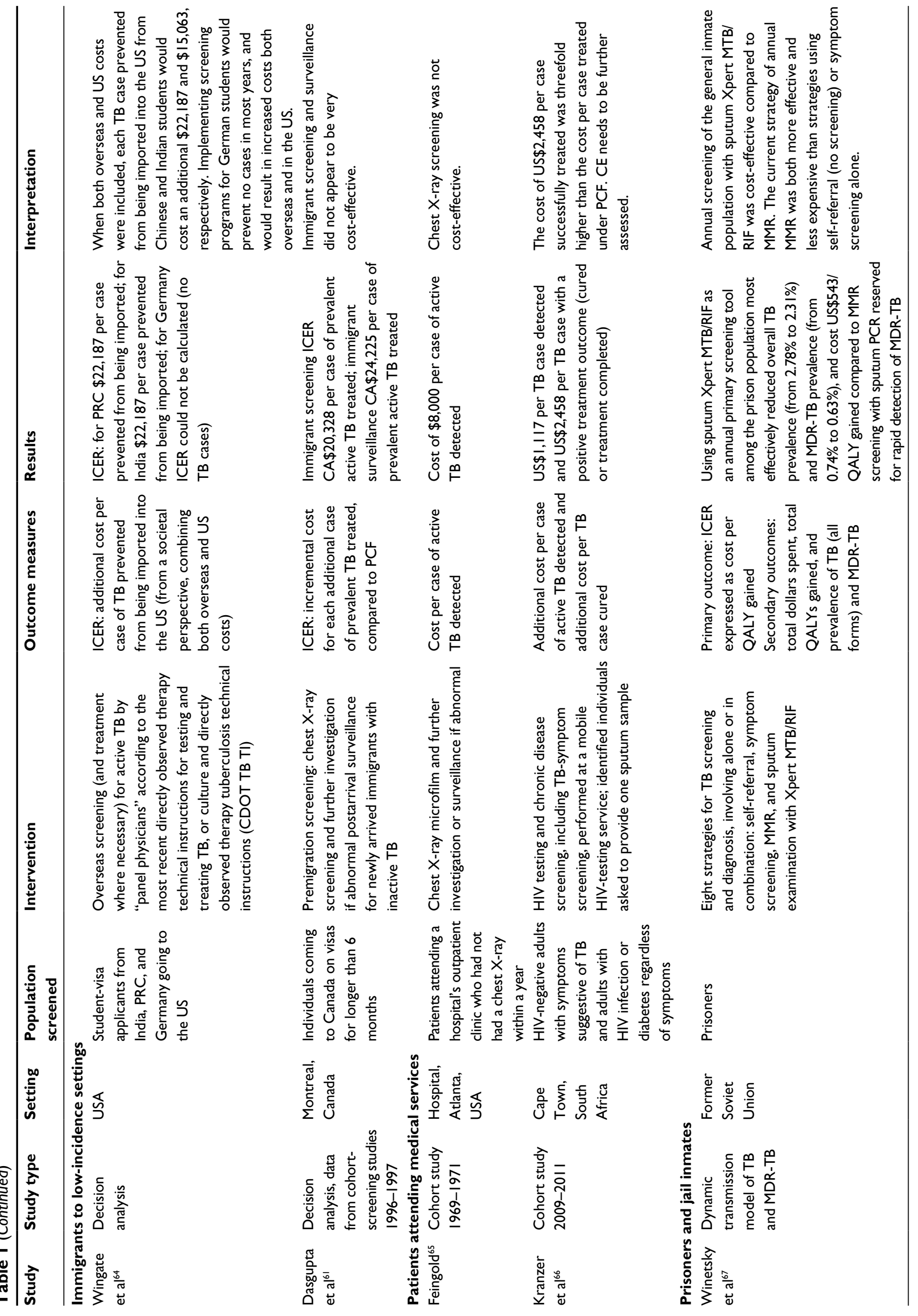




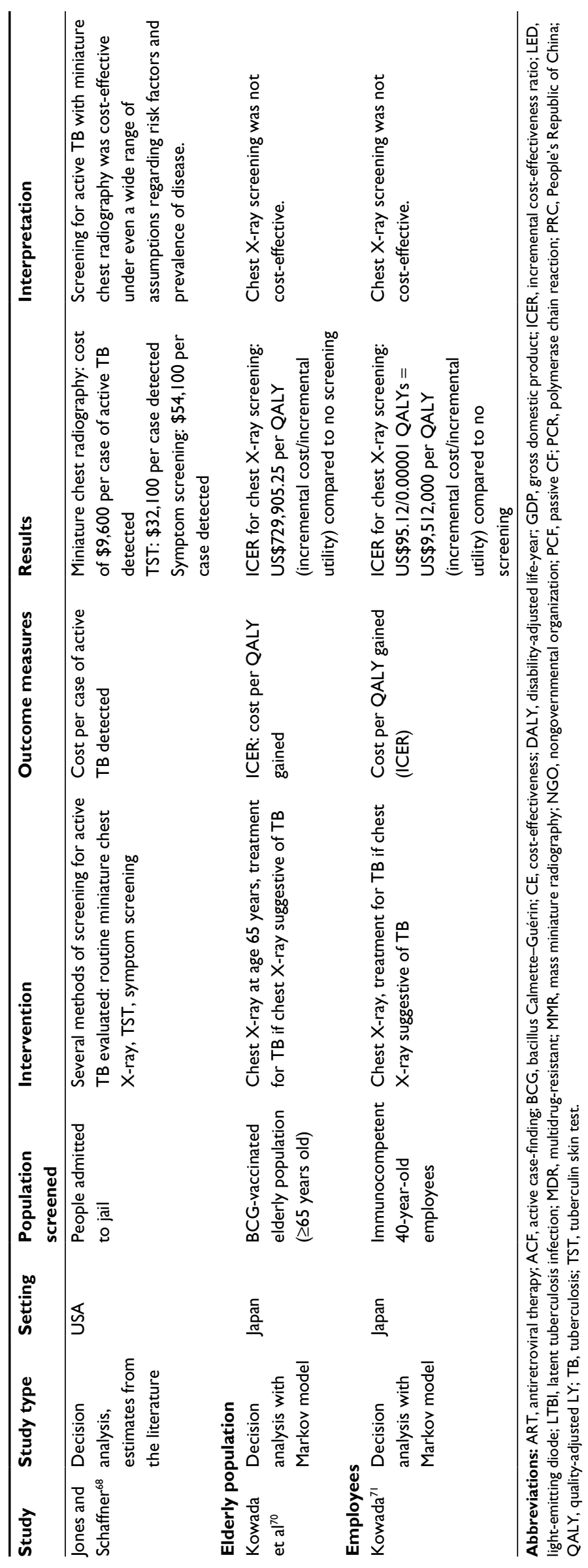

ClinicoEconomics and Outcomes Research 2016:8 
additional cost per TB case detected via screening without outlining the estimated difference in the effect compared to no screening or another screening method (PCF)..$^{59,65,66,68}$ Comparison with the effect of "no screening" is important, because it cannot be assumed that every TB case diagnosed through a screening intervention for active TB is an additional case detected compared to "no screening"/PCF. It is likely that some of these cases would eventually have been diagnosed with PCF when patients sought health care.

\section{Community-wide screening}

Of four studies evaluating costs for community-wide ACF, three performed a true $\mathrm{CEA}^{56-58}$ and one evaluated the incremental cost per TB case detected without estimating the incremental effect compared to PCF. ${ }^{59}$ Two studies examining ACF in Kampala, Uganda came to different conclusions regarding the cost-effectiveness of door-to-door screening with sputum collection in people who had had cough for 2 weeks or longer. ${ }^{56,58}$ This difference was despite the fact that both used data from the same pilot ACF survey in Kampala. ${ }^{72}$

Mupere et al calculated an ICER expressing cost per additional QALY, which was US\$109 and as such below the amount of GDP per capita (\$350) and highly cost-effective. ${ }^{58}$ Sekandi et al calculated an ICER expressing cost per additional TB case detected, which was US $\$ 1,493 .{ }^{56}$ As the study authors defined the cost-effectiveness threshold for this study at below twice Uganda's GDP per capita for the study period $(\$ 1,102)$, the cost did not fall below the threshold. The cost would, however, have been less than three times the national annual GDP per capita $(\$ 1,653)$, the cost-effectiveness threshold promoted by WHO-CHOICE. It needs to be noted that the cost-effectiveness threshold recommendations by WHO-CHOICE are based on cost per DALY averted, and cannot simply be adapted for nonstandardized effect measures, such as cost per TB case detected.

A mathematical modeling study estimated the costeffectiveness of discrete ACF campaigns lasting 2 years and of programmatic changes incorporating ACF into routine TB-control activities in the long term (study period 10 years) in India, the People's Republic of China, and South Africa. ${ }^{57}$ Discrete campaigns were all highly cost-effective (cost per DALY averted less than per capita GDP); prolonged integration of ACF was even more cost-effective. A study from Nepal evaluated the cost of ACF in the community using an outreach TB-diagnostic service (microscopy camps lasting 2-4 days) with precamp publicity. ${ }^{59}$ The cost was US $\$ 37.50$ per smearpositive diagnosed TB case, which was prohibitively expensive for the national health program when the entire ministry of health expenditure on health for the people of Nepal per annum was only $\sim \$ 1.30$ per person per year during the study period. The additional estimated cost for an international nongovernmental organization to run 15 camps a year, however, was only an estimated $0.35 \%$ of the overall running costs.

\section{Targeted screening of high-risk groups}

Three studies from Cambodia, Uganda, and Canada found that ACF among contacts of patients with contagious TB was cost-effective or even highly cost-effective. ${ }^{56,60,61}$ The ICER in the studies from Uganda and Canada was expressed as cost/savings for each additional case of active TB detected and each additional case of active TB treated, respectively. The ICER in the Cambodian study was expressed as cost per DALY averted (US\$330) and cost per death averted $(\$ 5,300)$, and was consistent with high cost-effectiveness (cost/DALY averted well below the GDP per capita). The Cambodian study used data from a large ACF program initiated in 2012 targeting household and symptomatic neighborhood contacts of known TB cases, covering more than a third of the population.

Two studies evaluated the cost-effectiveness of ACF among people with HIV infection in Africa. ${ }^{62,63}$ A CEA by Zwerling et al was based on data from a cluster-randomized trial of point-of-care screening for TB among people receiving a new HIV diagnosis in rural Malawi. ${ }^{62}$ It evaluated TB screening in all patients diagnosed with HIV and at least one TB symptom with Xpert MTB/RIF or light-emitting diode microscopy versus standard care (screening performed at the discretion of the treating physician with standard smear microscopy). Based on low patient volume, the ICER was US\$1,808/DALY averted for light-emitting diode microscopy and US\$699/DALY averted for Xpert MTB/RIF. Using a cost-effectiveness threshold of three times the GDP per capita for Malawi $(\$ 1,080)$, the ICER was higher than the cost-effectiveness threshold; however, screening for TB with Xpert MTB/RIF would have been cost-effective in most other sub-Saharan low-income countries. A South African study found that screening all HIV-infected individuals initiating antiretroviral therapy with two Xpert MTB/RIF tests was highly cost-effective. ${ }^{63}$ Both African studies found that the prevalence of active TB was a key driver of cost-effectiveness when considering TB screening for people with newly diagnosed HIV, with the study in Malawi additionally identifying test volume as a key driver of cost-effectiveness.

A Canadian study found that TB screening and surveillance in immigrants was not very cost-effective, with an ICER of CA $\$ 20,328$ per case of prevalent active TB treated 
for the screening program and an ICER of \$24,225 per case of prevalent active TB treated for the surveillance program (both compared to PCF) ${ }^{61}$ The study authors explained the lack of cost-effectiveness mainly with operational problems of the screening/surveillance program. Another study pointed out that premigration screening can save the destination country significant amounts of money if screening overseas is performed at the expense of the visa applicants. ${ }^{64}$

The value of screening patients attending medical services for active TB was assessed in two studies, but neither calculated an ICER. ${ }^{65,66}$ A study conducted in the US from 1969 to 1971 found that chest X-ray screening of patients attending outpatient clinics in a US hospital was not costeffective. A South African study evaluated the cost of screening HIV-negative adults with symptoms suggestive of TB and all adults with HIV infection or diabetes regardless of symptoms who attended a mobile HIV-testing service. ${ }^{66}$ The cost of US $\$ 2,458$ per case treated successfully was threefold higher than the cost per case treated under PCF in this study, suggesting that ACF may not be cost-effective.

A study using a dynamic transmission model for TB and MDR-TB found that annual screening with Xpert MTB/RIF of prisoners in the former Soviet Union most effectively reduced TB and MDR-TB and was cost-effective. ${ }^{67}$ The current strategy of annual MMR was both more effective and less expensive than strategies using self-referral or symptom screening alone. Another study found that miniature chest $\mathrm{X}$-ray screening of jail inmates in the US was more costeffective than TST and symptom screening, but the costeffectiveness of miniature chest X-ray screening compared to no screening was not assessed. ${ }^{68}$

A Japanese study found that chest X-ray screening for active TB among the elderly (65-year-old bacillus CalmetteGuérin-vaccinated persons) was not cost-effective (ICER of US\$729,905.25 per QALY compared to no screening). ${ }^{70}$ Another Japanese study found that chest X-ray screening among immunocompetent 40-year-old employees was not cost-effective (ICER of US\$9,512,000 per QALY compared to no screening). ${ }^{71}$

\section{Conclusion}

There are a number of relatively recent CEAs that have focused on screening and diagnostic tests for LTBI, but there are few studies that have evaluated the cost-effectiveness of screening for active TB. CEAs of community-wide ACF suggest that this intervention can be cost-effective or even highly cost-effective in settings with a high incidence of TB. ACF among (close) TB contacts was found to be (highly) cost-effective in settings with a low as well as a high incidence of TB. The evidence for cost-effectiveness of screening among HIV-infected persons is not as strong as for TB contacts, but the reviewed studies suggest that the intervention can be (highly) cost-effective depending on the background prevalence of TB and test volume. Screening of prisoners and jail inmates was cost-effective in studies from the US and the former Soviet Union. Screening and surveillance programs for TB among migrants funded by destination countries with a low incidence of TB were not cost-effective.

The evidence from all analyzed CEAs is severely limited by the fact that none of the estimates for incremental effectiveness (compared to PCF) was based on randomized controlled trials. Randomized controlled trials that compare ACF with PCF in different populations are thus urgently needed to inform CEAs. New TB-diagnostic tests impact on the cost-effectiveness of screening, and integrating these tools into trials on ACF strategies is essential. As the results of randomized controlled trials evaluating different ACF strategies will become available in future, we will hopefully gain a better understanding of the role that ACF can play in achieving global TB control.

\section{Disclosure}

CCD was supported by a TRIP (Translating Research Into Practice) Fellowship from the Australian National Health and Medical Research Council (NHMRC). The funders of the fellowship had no role in study design, data collection and analysis, decision to publish, or preparation of the manuscript. The author reports no conflicts of interest in this work.

\section{References}

1. World Health Organization. Global Tuberculosis Report 2014. Geneva: WHO; 2014.

2. World Health Organization. Global strategy and targets for tuberculosis prevention, care and control after 2015. 2014. Available from: http:// www.who.int/tb/post2015_strategy/en. Accessed May 25, 2016.

3. Pinet G. Good Practice in Legislation and Regulations for TB Control: An Indicator of Political Will. Geneva: WHO; 2001.

4. Abebe M, Doherty M, Wassie L, et al. TB case detection: can we remain passive while the process is active? Pan Afr Med J. 2012;11:50.

5. Dowdy DW, Chaisson RE. The persistence of tuberculosis in the age of DOTS: reassessing the effect of case detection. Bull World Health Organ. 2009;87(4):296-304.

6. Dudley SF. Pulmonary tuberculosis in the Royal Navy and the use of mass miniature radiography in its control. Proc R Soc Med. 1941;34(7): 401-406.

7. Wayburn E. Mass miniature radiography; a survey in the United States Army Air Forces. Am Rev Tuberc. 1946;54(6):527-540.

8. Dick WP. Mass miniature radiography of factory groups. $\mathrm{Br} \mathrm{Med} J$ 1945;2(4425):568-569. 
9. Bynum H. Spitting Blood: The History of Tuberculosis. Oxford: Oxford University Press; 2012.

10. Raviglione MC, Pio A. Evolution of WHO policies for tuberculosis control, 1948-2001. Lancet. 2002;359(9308):775-780.

11. Golub JE, Mohan CI, Comstock GW, Chaisson RE. Active case finding of tuberculosis: historical perspective and future prospects. Int JTuberc Lung Dis. 2005;9(11):1183-1203.

12. Banerji D, Andersen S. A sociological study of awareness of symptoms among persons with pulmonary tuberculosis. Bull World Health Organ. 1963;29(5):665-683.

13. Baily GV, Savic D, Gothi GD, Naidu VB, Nair SS. Potential yield of pulmonary tuberculosis cases by direct microscopy of sputum in a district of south India. Bull World Health Organ. 1967;37(6):875-892.

14. [No authors listed]. Tuberculosis baseline survey in Burma in 1972. Tubercle. 1974;55(4):313-325.

15. Santha T, Garg R, Subramani R, et al. Comparison of cough of 2 and 3 weeks to improve detection of smear-positive tuberculosis cases among out-patients in India. Int J Tuberc Lung Dis. 2005;9(1):61-68.

16. Stýblo K, Danková D, Drápela J, et al. Epidemiological and clinical study of tuberculosis in the district of Kolin, Czechoslovakia: report for the first 4 years of the study (1961-64). Bull World Health Organ. 1967;37(6):819-874.

17. Krivinka R, Drápela J, Kubík A, et al. Epidemiological and clinical study of tuberculosis in the district of Kolin, Czechoslovakia: second report (1965-1972). Bull World Health Organ. 1974;51(1):59-69.

18. Stýblo K. Epidemiology of Tuberculosis. 2nd ed. The Hague: Royal Netherlands Tuberculosis Association (KNCV); 1991.

19. Toman K. Mass radiography in tuberculosis control. WHO Chron. 1976;30(2):51-57.

20. World Health Organization. WHO Expert Committee on Tuberculosis: Ninth Report. Geneva: WHO; 1974.

21. Kranzer K, Afnan-Holmes H, Tomlin K, et al. The benefits to communities and individuals of screening for active tuberculosis disease: a systematic review. Int J Tuberc Lung Dis. 2013;17(4):432-446.

22. Shapiro A, Golub JE. Systematic review of number needed to screen (NNS) in selected risk groups with different screening approaches. Poster presented at: Scoping Meeting for the Development of Guidelines on Screening for Active TB; 31 May-1 June 2011; Geneva, Switzerland.

23. Shargie EB, Mørkve O, Lindtjørn B. Tuberculosis case-finding through a village outreach programme in a rural setting in southern Ethiopia: community randomized trial. Bull World Health Organ. 2006;84(2):112-119.

24. Datiko DG, Lindtjørn B. Health extension workers improve tuberculosis case detection and treatment success in southern Ethiopia: a community randomized trial. PLoS One. 2009;4(5):e5443.

25. Moyo S, Verver S, Hawkridge A, et al. Tuberculosis case finding for vaccine trials in young children in high-incidence settings: a randomised trial. Int J Tuberc Lung Dis. 2012;16(2):185-191.

26. Miller AC, Golub JE, Cavalcante SC, et al. Controlled trial of active tuberculosis case finding in a Brazilian favela. Int J Tuberc Lung Dis. 2010;14(6):720-726.

27. Corbett EL, Bandason T, Duong T, et al. Comparison of two active case-finding strategies for community-based diagnosis of symptomatic smear-positive tuberculosis and control of infectious tuberculosis in Harare, Zimbabwe (DETECTB): a cluster-randomised trial. Lancet. 2010;376(9748):1244-1253.

28. Okada K, Onozaki I, Yamada N, et al. Epidemiological impact of mass tuberculosis screening: a 2-year follow-up after a national prevalence survey. Int J Tuberc Lung Dis. 2012;16(12):1619-1624.

29. Cavalcante SC, Durovni B, Barnes GL, et al. Community-randomized trial of enhanced DOTS for tuberculosis control in Rio de Janeiro, Brazil. Int J Tuberc Lung Dis. 2010;14(2):203-209.

30. Rendleman NJ. Mandated tuberculosis screening in a community of homeless people. Am J Prev Med. 1999;17(2):108-113.

31. Ayles H, Muyoyeta M, Du Toit E, et al. Effect of household and community interventions on the burden of tuberculosis in southern Africa: the ZAMSTAR community-randomised trial. Lancet. 2013;382(9899):1183-1194.
32. World Health Organization. Systematic Screening for Active Tuberculosis: Principles and Recommendations. Geneva: WHO; 2013.

33. Fox GJ, Dobler CC, Marks GB. Active case finding in contacts of people with tuberculosis. Cochrane Database Syst Rev. 2011;(9): CD008477.

34. Shah L, Rojas M, Mori O, et al. Implementation of a stepped-wedge cluster randomized design in routine public health practice: design and application for a tuberculosis (TB) household contact study in a high burden area of Lima, Peru. BMC Public Health. 2015;15:587.

35. Fox GJ, Nhung NV, Sy DN, Britton WJ, Marks GB. Household contact investigation for tuberculosis in Vietnam: study protocol for a cluster randomized controlled trial. Trials. 2013;14:342.

36. Banoo S, Bell D, Bossuyt P, et al. Evaluation of diagnostic tests for infectious diseases: general principles. Nat Rev Microbiol. 2006;4(12 Suppl):S20-S32.

37. Pai M, Minion J, Sohn H, Zwerling A, Perkins MD. Novel and improved technologies for tuberculosis diagnosis: progress and challenges. Clin Chest Med. 2009;30(4):701-716, viii.

38. Steingart KR, Ramsay A, Pai M. Optimizing sputum smear microscopy for the diagnosis of pulmonary tuberculosis. Expert Rev Anti Infect Ther. 2007;5(3):327-331.

39. Nicol MP, Zar HJ. New specimens and laboratory diagnostics for childhood pulmonary TB: progress and prospects. Paediatr Respir Rev. 2011;12(1):16-21.

40. Reid MJ, Shah NS. Approaches to tuberculosis screening and diagnosis in people with HIV in resource-limited settings. Lancet Infect Dis. 2009;9(3):173-184.

41. Rawat J, Biswas D, Sindhwani G, Kesharwani V, Masih V, Chauhan BS. Diagnostic defaulters: an overlooked aspect in the Indian Revised National Tuberculosis Control Program. J Infect Dev Ctries. 2012;6(1): 20-22.

42. Boehme CC, Nicol MP, Nabeta P, et al. Feasibility, diagnostic accuracy, and effectiveness of decentralised use of the Xpert MTB/RIF test for diagnosis of tuberculosis and multidrug resistance: a multicentre implementation study. Lancet. 2011;377(9776):1495-1505.

43. Singh KK, Muralidhar M, Kumar A, et al. Comparison of in house polymerase chain reaction with conventional techniques for the detection of Mycobacterium tuberculosis DNA in granulomatous lymphadenopathy. J Clin Pathol. 2000;53(5):355-361.

44. Pai M, Schito M. Tuberculosis diagnostics in 2015: landscape, priorities, needs, and prospects. J Infect Dis. 2015;211(Supp1 2):S21-S28.

45. Steingart KR, Schiller I, Horne DJ, Pai M, Boehme CC, Dendukuri N. Xpert(R) MTB/RIF assay for pulmonary tuberculosis and rifampicin resistance in adults. Cochrane Database Syst Rev. 2014;1:CD009593.

46. World Health Organization. WHO monitoring of Xpert MTB/RIF roll-out. 2013. Available from: http://www.who.int/tb/areas-of-work/ laboratory/mtb-rif-rollout/en. Accessed May 26, 2016.

47. UNITAID. Tuberculosis: Diagnostics Technology and Market Landscape. 3rd ed. Geneva: World Health Organization; 2014.

48. Pai M. Innovations in tuberculosis diagnostics: progress and translational challenges. EBioMedicine. 2015;2(3):182-183.

49. [No authors listed]. Diagnostic standards and classification of tuberculosis. Am Rev Respir Dis. 1990;142(3):725-735.

50. Pai M, Rodrigues C. Management of latent tuberculosis infection: an evidence-based approach. Lung India. 2015;32(3):205-207.

51. Pai M, Gokhale K, Joshi R, et al. Mycobacterium tuberculosis infection in health care workers in rural India: comparison of a whole-blood interferon $\gamma$ assay with tuberculin skin testing. JAMA. 2005;293(22): 2746-2755.

52. World Health Organization. The Global Burden of Disease concept. Available from: http:/www.who.int/quantifying_ehimpacts/publications/ en/9241546204chap3.pdf. Accessed March 13, 2016.

53. Tan Torres T, Baltussen R,Adam T, et al, editors. Making Choices in Health: WHO Guide to Cost-Effectiveness Analysis. Geneva: World Health Organization; 2003. Available from: http:/www.who.int/choice/publications/ p_2003_generalised_cea.pdf. Accessed March 13, 2016.

54. World Health Organization. Macroeconomics and Health: Investing in Health for Economic Development. Geneva: WHO; 2001. 
55. Hutubessy R, Chisholm D, Edejer TT. Generalized cost-effectiveness analysis for national-level priority-setting in the health sector. Cost Eff Resour Alloc. 2003;1(1):1-13.

56. Sekandi JN, Dobbin K, Oloya J, Okwera A, Whalen CC, Corso PS. Costeffectiveness analysis of community active case finding and household contact investigation for tuberculosis case detection in urban Africa. PLoS One. 2015;10(2):e0117009.

57. Azman AS, Golub JE, Dowdy DW. How much is tuberculosis screening worth? Estimating the value of active case finding for tuberculosis in South Africa, China, and India. BMC Med. 2014;12:216.

58. Mupere E, Schiltz NK, Mulogo E, Katamba A, Nabbuye-Sekandi J, Singer ME. Effectiveness of active case-finding strategies in tuberculosis control in Kampala, Uganda. Int JTuberc Lung Dis. 2013;17(2):207-213.

59. Harper I, Fryatt R, White A. Tuberculosis case finding in remote mountainous areas: are microscopy camps of any value? Experience from Nepal. Tuber Lung Dis. 1996;77(4):384-388.

60. Yadav RP, Nishikiori N, Satha P, Eang MT, Lubell Y. Cost-effectiveness of a tuberculosis active case finding program targeting household and neighborhood contacts in Cambodia. Am JTrop Med Hyg. 2014;90(5):866-872.

61. Dasgupta K, Schwartzman K, Marchand R, Tennenbaum TN, Brassard P, Menzies D. Comparison of cost-effectiveness of tuberculosis screening of close contacts and foreign-born populations. Am J Respir Crit Care Med. 2000;162(6):2079-2086.

62. Zwerling AA, Sahu M, Ngwira LG, et al. Screening for tuberculosis among adults newly diagnosed with HIV in sub-Saharan Africa: a cost-effectiveness analysis. J Acquir Immune Defic Syndr. 2015;70(1): 83-90.

63. Andrews JR, Lawn SD, Rusu C, et al. The cost-effectiveness of routine tuberculosis screening with Xpert MTB/RIF prior to initiation of antiretroviral therapy: a model-based analysis. AIDS. 2012;26(8):987-995.
64. Wingate LT, Coleman MS, Posey DL, et al. Cost-effectiveness of screening and treating foreign-born students for tuberculosis before entering the United States. PLoS One. 2015;10(4):e0124116.

65. Feingold AO. Cost effectiveness of screening for tuberculosis in a general medical clinic. Public Health Rep. 1975;90(6):544-547.

66. Kranzer K, Lawn SD, Meyer-Rath G, et al. Feasibility, yield, and cost of active tuberculosis case finding linked to a mobile HIV service in Cape Town, South Africa: a cross-sectional study. PLoS Med. 2012;9(8):e1001281.

67. Winetsky DE, Negoescu DM, DeMarchis EH, et al. Screening and rapid molecular diagnosis of tuberculosis in prisons in Russia and Eastern Europe: a cost-effectiveness analysis. PLoS Med. 2012;9(11) e1001348.

68. Jones TF, Schaffner W. Miniature chest radiograph screening for tuberculosis in jails: a cost-effectiveness analysis. Am J Respir Crit Care Med. 2001;164(1):77-81.

69. Kowada A. Cost effectiveness of interferon-gamma release assay for tuberculosis screening of rheumatoid arthritis patients prior to initiation of tumor necrosis factor- $\alpha$ antagonist therapy. Mol Diagn Ther. 2010;14(6):367-373.

70. Kowada A, Deshpande GA, Takahashi O, Shimbo T, Fukui T. Cost effectiveness of interferon-gamma release assay versus chest X-ray for tuberculosis screening of BCG-vaccinated elderly populations. $\mathrm{Mol}$ Diagn Ther. 2010;14(4):229-236.

71. Kowada A. Cost-effectiveness of interferon- $\gamma$ release assay versus chest X-ray for tuberculosis screening of employees. Am J Infect Control. 2011;39(10):e67-e72.

72. Sekandi JN, Neuhauser D, Smyth K, Whalen CC. Active case finding of undetected tuberculosis among chronic coughers in a slum setting in Kampala, Uganda. Int J Tuberc Lung Dis. 2009;13(4):508-513.
ClinicoEconomics and Outcomes Research

\section{Publish your work in this journal}

ClinicoEconomics and Outcomes Research is an international, peerreviewed open-access journal focusing on health technology assessment, pharmacoeconomics and outcomes research in the areas of diagnosis, medical devices, and clinical, surgical and pharmacological intervention. The economic impact of health policy and health systems
Submit your manuscript here: https://www.dovepress.com/clinicoeconomics-and-outcomes-reserch-jur

organization also constitute important areas of coverage. The manuscript management system is completely online and includes a very quick and fair peer-review system, which is all easy to use. Visit http://www.dovepress.com/testimonials.php to read real quotes from published authors. 